\title{
ㄷำ1
}

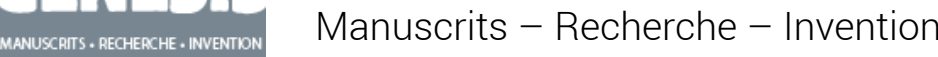

39 | 2014

Avant-dire

\section{Cinthia Meli - Bossuet : currente calamo, viva voce}

Entretien avec Gilles Philippe

\section{Gilles Philippe}

\section{(2) OpenEdition}

\section{Journals}

Édition électronique

URL : http://journals.openedition.org/genesis/1390

DOI : $10.4000 /$ genesis. 1390

ISSN : 2268-1590

Éditeur :

Presses universitaires de Paris Sorbonne (PUPS), Société internationale de génétique artistique littéraire et scientifique (SIGALES)

\section{Édition imprimée}

Date de publication : 17 novembre 2014

Pagination : 87-100

ISBN : 9782840509714

ISSN : $1167-5101$

\section{Référence électronique}

Gilles Philippe, «Cinthia Meli - Bossuet : currente calamo, viva voce», Genesis [En ligne], 39 | 2014, mis en ligne le 22 décembre 2016, consulté le 19 avril 2019. URL : http://journals.openedition.org/ genesis/1390; DOl : 10.4000/genesis.1390 


\title{
Cinthia Meli \\ Bossuet : currente calamo, viva voce
}

\author{
Entretien avec Gilles Philippe
}

Gilles Philippe - Cinthia Meli, vous avez soutenu en janvier 2012 à l'université de Genève une thèse de doctorat consacrée aux pratiques d'écriture et de publication de Bossuet ${ }^{1}$, dans laquelle vous vous penchez notamment sur les manuscrits autographes de ses sermons selon une perspective proche de celle que nous avons adoptée pour ce numéro de Genesis. Pouvez-vous nous expliquer comment vous en êtes arrivée à vous interroger sur les liens entre écriture et oralité au sujet de ce corpus ? Et d'abord, d'où vous vient cet intérêt pour un genre aussi peu canonique que le sermon?

Cinthia Meli - Le genre du sermon n'est peut-être plus très canonique aujourd'hui, mais il l'était, au XVIII' siècle, au moment où l'idée même de littérature est en train d'émerger : à ce titre, il me paraissait digne d'être étudié. J'avais alors l'intention de fonder ma thèse sur une analyse des procédés rhétoriques et stylistiques à l'œuvre dans les sermons de Bossuet, une analyse qui tiendrait compte de leurs circonstances de performance initiales pour tenter d'en saisir l'efficacité : j'étais intéressée en effet par le caractère événementiel de ces discours et par les effets qu'ils avaient pu avoir sur leurs auditeurs originels. Cette efficacité était célébrée notamment par l'anecdote du Tu es ille vir, rapportée par Constance Cagnat-Debœuf dans l'édition «Folio » du Carême $d u$ Louvre (2001), selon laquelle Louis XIV aurait baissé la tête face à une accusation d'adultère à peine voilée lancée en chaire par Bossuet. Un tel projet supposait évidemment que le texte des Euvres oratoires fût identique aux paroles prononcées par le prédicateur, une hypothèse qui semblait alors largement partagée par la critique, même si elle était rarement explicitée et jamais discutée : le rapport entre texte et discours constituait un point aveugle des études consacrées à Bossuet en 2003, lorsque le Carême du Louvre a été mis au programme de l'agrégation. La question m'est apparue dès lors essentielle, puisqu'elle mettait au fond en jeu l'intérêt, voire la validité des analyses dont ce texte pouvait faire l'objet par la critique.

Comment avez-vous procédé pour résoudre une telle question? Quelle a été votre démarche?

J'ai commencé simplement par m'interroger sur la qualité et donc sur les principes de l'édition de référence des Euvres oratoires de Bossuet, donnée par Joseph Lebarq à la fin du $\mathrm{XIX}^{\mathrm{e}}$ siècle, puis révisée et augmentée par Charles Urbain et Eugène Levesque au début du $\mathrm{xx}^{\mathrm{e}}$ siècle. J'ai découvert qu'elle constituait en fait l'aboutissement d'un processus éditorial de longue haleine, entrepris dans le dernier tiers du XVIII siècle, puis poursuivi dans la seconde moitié du $\mathrm{XIX}^{\mathrm{e}}$ siècle sous l'impulsion de Victor Cousin et du développement des études philologiques en France. Le processus a impliqué une dizaine d'intervenants et s'est traduit par quatre études historiques et philologiques et une dizaine d'éditions partielles ou complètes des sermons. Il faut dire qu'à l'exception de sept oraisons

1. Le Livre et la Chaire, à paraître en 2014 aux éditions Honoré Champion. 


\section{2}

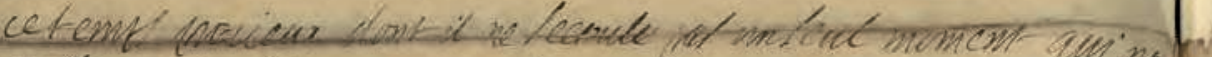

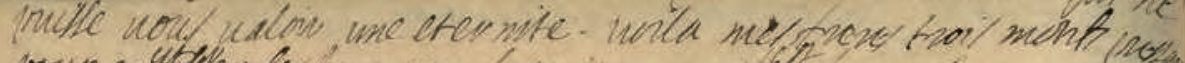

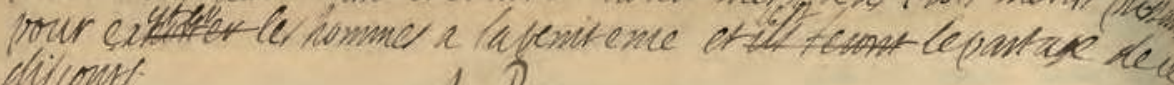
Ailcont.<smiles>[Te]=[Te]</smiles>

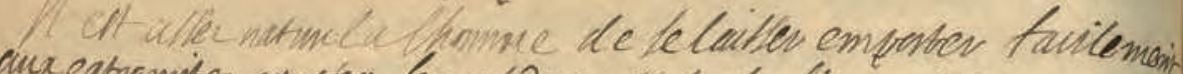

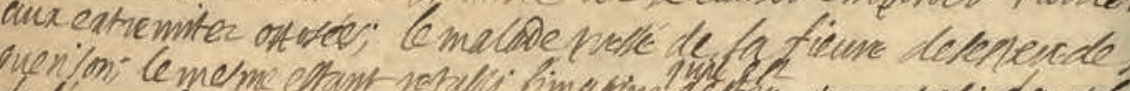

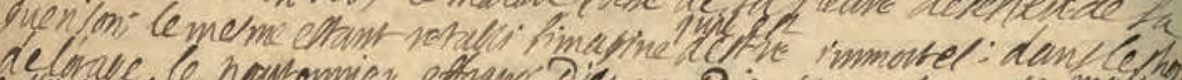

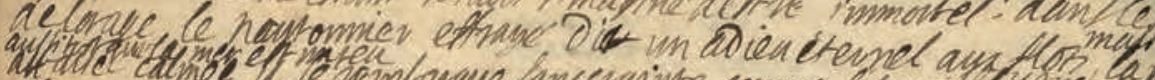

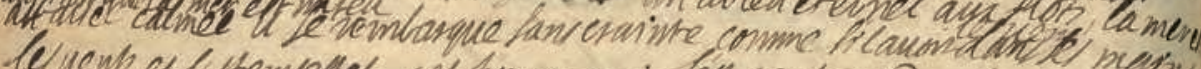

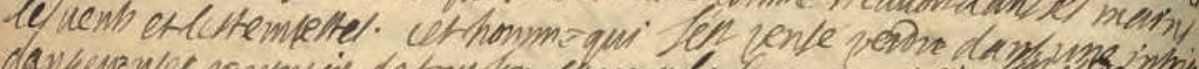

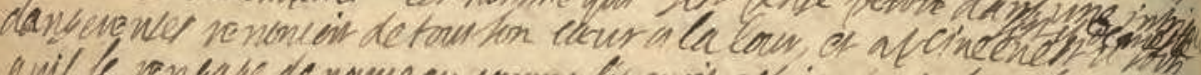

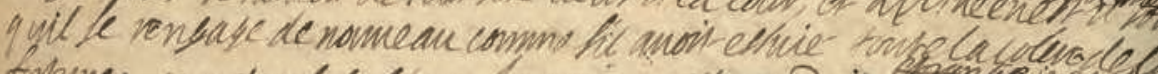

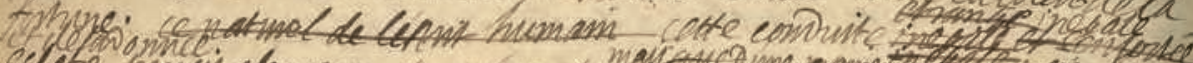

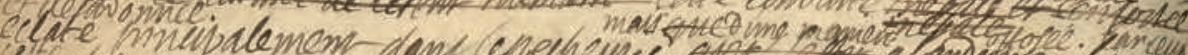

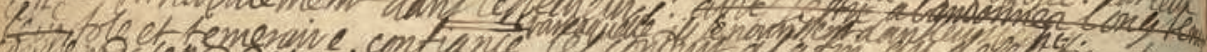

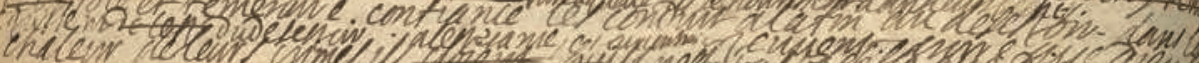

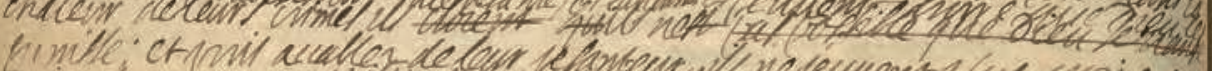
sicu cur (1)

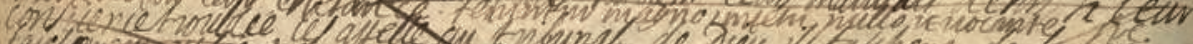

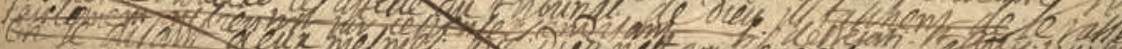

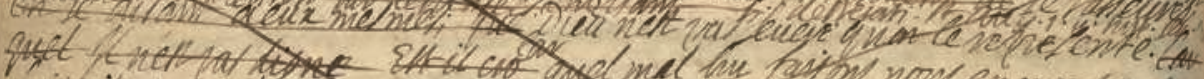

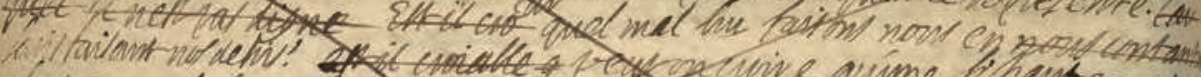

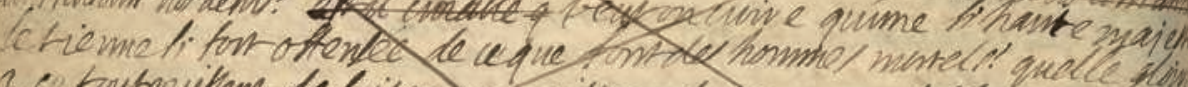

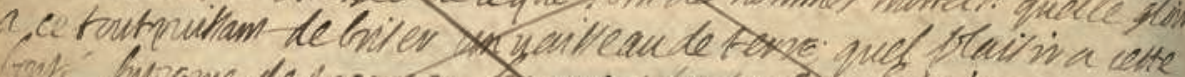

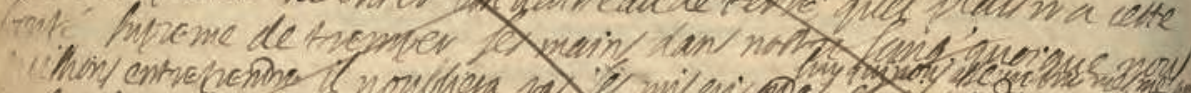

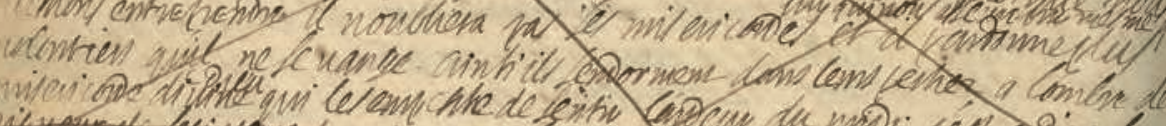

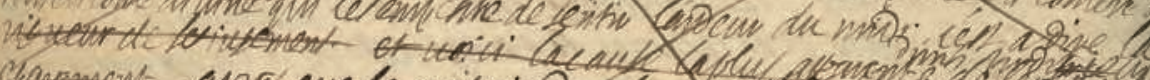

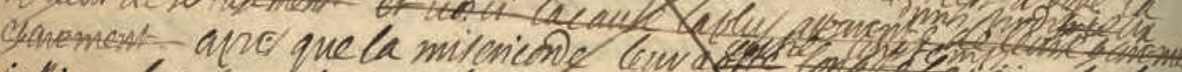

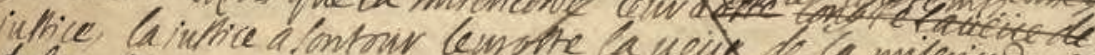

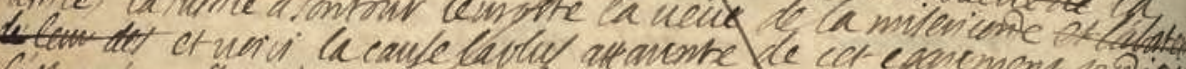

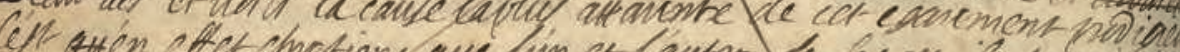

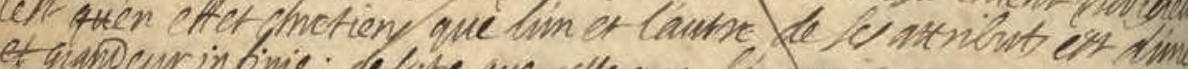

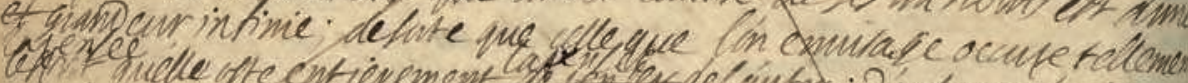

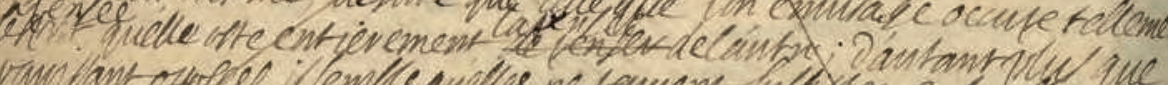

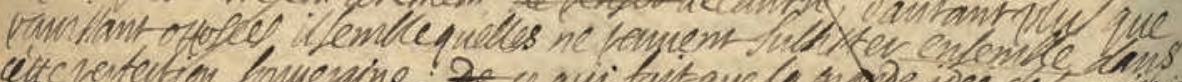

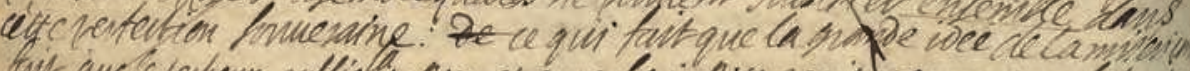

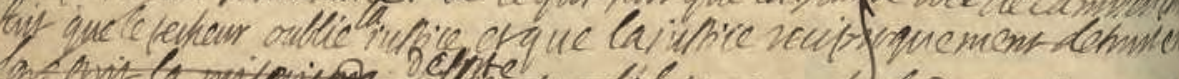

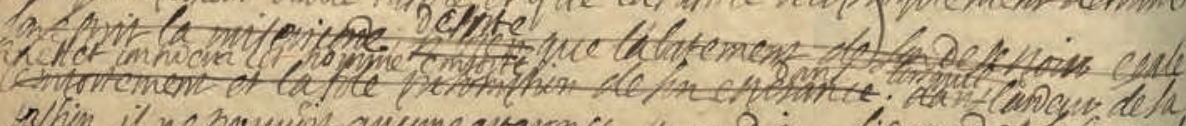

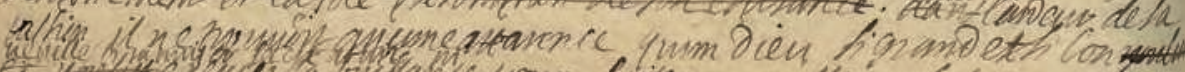

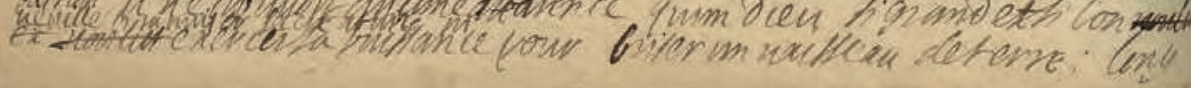

Fig. 1 : Bossuet, Sermon sur la pénitence, Carême des Carmélites (1661), f. 87 v (BnF, Fonds français 12822) 
funèbres et de deux sermons, les Euvres oratoires de Bossuet n'ont été publiées qu'à titre posthume, suite à la découverte, dans les années 1760, d'un ensemble très important de manuscrits autographes, qui contenait les notes ou les textes de près de deux cents discours. Or ces manuscrits ont posé à leurs éditeurs de sérieuses difficultés, qui expliquent qu'il ait fallu attendre près de cent cinquante ans pour parvenir à une édition jugée définitive.

\section{De quel ordre étaient ces difficultés?}

Une première série de difficultés tenait à l'établissement du texte proprement dit, puisque les manuscrits, d'une écriture parfois difficile à déchiffrer, pas toujours entièrement rédigés, et comportant dans certains cas des corrections currente calamo, présentent en outre des variantes (plusieurs termes équivalents sont superposés au-dessus d'un mot sans que Bossuet ait marqué sa préférence), des indications de corrections à faire laissées en suspens (typiquement la mention marginale « abréger ») ou difficiles à interpréter (comme le soulignement, qui peut indiquer indifféremment l'importance d'un passage ou l'intention de le supprimer). Une seconde difficulté était liée à l'identification de chaque discours : Bossuet est accoutumé en effet à la pratique de la reprise (qui consiste en la copie d'un passage d'un sermon antérieur dans un sermon en cours de composition, avec dans certains cas l'introduction de corrections), une pratique qui se traduit parfois par un remploi matériel (les pages d'un sermon antérieur sont transférées dans le nouveau sermon), qui entraîne sur le manuscrit une double, voire une triple numérotation des pages. Enfin une dernière difficulté consistait à attribuer à chaque discours sa date de composition : si les sermons des stations de carême et d'avent portent des traces de classement qui permettent d'en déduire la date probable (du type : «Car. Min. 3 dim. » pour le troisième dimanche du Carême des Minimes de 1660), les autres ne comportent jamais la moindre indication, et ils ont fait parfois l'objet de reprises intégrales (le même sermon est prononcé à diverses occasions, parfois à des années d'écart, Bossuet apportant dans certains cas des corrections au manuscrit).
Comment les éditeurs des XVIII et XIXe siècles ont-ils résolu ces difficultés?

En s'appuyant sur une expertise philologique faillible et toujours à conquérir (en témoignent les lectures divergentes que les éditeurs font de certains mots, données comme variantes dans les éditions ultérieures, et les désaccords qu'ils ont sur les méthodes de datation des sermons, au reste toutes douteuses), en procédant à des interventions qui semblent excéder leur mission (le premier éditeur, Deforis, rédige les passages laissés à l'état de notes par Bossuet et fond deux sermons inachevés en un seul) et enfin en opérant des choix parfaitement arbitraires (un autre éditeur, Lachat, privilégie une variante parmi un choix de variantes non tracées ou conserve un passage pourtant expressément barré par Bossuet). Si l'on pousse la réflexion jusqu'au bout, les Euvres oratoires publiées par Urbain et Levesque sont au fond autant l'œuvre de Bossuet que celle de ses éditeurs successifs : c'est moins une édition définitive qu'une édition conventionnelle.

\section{Pourquoi n'avoir pas procédé dans ce cas à une nouvelle édition?}

D'abord parce que je n'en avais ni les compétences paléographiques, ni les possibilités matérielles ; ensuite parce que, quand bien même la science de l'édition couplée à l'outil informatique permettrait sans doute de surmonter les difficultés initiales posées par les manuscrits de Bossuet, l'état dans lequel ils sont à ce jour rend délicat un tel projet : sans mentionner les pièces disparues (une partie des manuscrits édités au XVIII et au XIXe siècle appartenait à des collections privées qui ont été entre-temps dispersées), les manuscrits conservés à la Bibliothèque nationale portent la trace des différentes manipulations dont ils ont fait l'objet. Certains ont été annotés par les éditeurs (notamment par le premier, Deforis, dont l'écriture n'est pas toujours facile à distinguer de celle de Bossuet, et par le dernier, Lebarq) et l'ensemble du corpus a été relié à la fin du XIXe siècle dans cinq volumes in-folio et classé en fonction de la première édition des sermons, selon l'ordre du calendrier liturgique : il paraît difficile, dans ces conditions, de reprendre le travail à zéro, en faisant table 
rase de toutes les interventions passées. Plutôt que d'ajouter un nouveau chapitre à cette entreprise éditoriale, j'ai préféré en faire l'histoire, en m'attachant à mettre au jour les difficultés qu'elle avait posées et les modèles auctoriaux et textuels qui la sous-tendaient et qui infléchissaient le travail des éditeurs.

\section{Quels sont ces modèles?}

Bossuet est envisagé par le premier éditeur, Deforis, comme un auteur ecclésiastique, doté d'un magistère théologique et moral, dont les textes, laissés à l'état de brouillons et négligés une fois sa mission accomplie, exigent d'être améliorés et complétés avant d'être donnés à méditer aux lecteurs, classés selon 1'ordre du calendrier liturgique. Au XIX ${ }^{\mathrm{e}}$ siècle, il est davantage perçu comme un écrivain français dont les brouillons montrent soit l'inspiration (ils sont inachevés), soit le métier (ils sont raturés) : leur publication en l'état, selon l'ordre chronologique, doit permettre aux amateurs de littérature de mesurer les progrès de son style et l'évolution de son œuvre. À cet égard, j'ai remarqué que les éditeurs du dernier tiers du XIX ${ }^{\mathrm{e}}$ siècle butent sur une difficulté, qui tient au rapport entre texte et œuvre, et qui croise la question qui me préoccupait, celle des rapports entre texte et discours. Pour les uns, l'œuvre de Bossuet correspond aux sermons qu'il a prononcés en chaire, et les manuscrits n'en constituent au fond que les brouillons préparatoires : l'œuvre est perdue, et ne laisse que des traces jugées au reste soit inférieures, soit supérieures à celle-ci. Pour les autres, les manuscrits peuvent être identifiés à l'œuvre, à condition de ramener celle-ci à une œuvre de nature rhétorique, où le contenu et l'articulation du propos importent plus que son expression, qu'elle soit écrite ou orale : dans ce cas, les sermons sont moins considérés comme des textes que comme des dispositifs discursifs. Mais quelle que soit leur prise de position en la matière, les éditeurs s'accordent sur un point : le discours prononcé en chaire par Bossuet diffère du texte saisi au manuscrit. Leur unanimité ne faisait évidemment pas mon affaire, mais elle était fondée sur le témoignage discutable du secrétaire de Bossuet, l'abbé Ledieu, qui niait la réalité matérielle (l'existence de sermons entièrement rédigés) pour prêter à son patron une technique d'improvisation fondée sur des canevas plus en phase avec le modèle de prédicateur apostolique qu'il cherchait à lui faire incarner. La question du lien entre texte manuscrit et discours performé restait donc posée.

\section{Qu'avez-vous alors fait?}

J'ai décidé de reprendre la question à nouveaux frais, en m'inspirant cette fois des méthodes et des perspectives de la critique génétique, qui souhaite depuis un certain temps déjà étendre ses enquêtes à des dossiers antérieurs à 1800 - pour autant que de tels dossiers existent. L'ensemble constitué par les manuscrits autographes de Bossuet est à cet égard tout à fait exceptionnel, mais il n'est pas sans poser problème à qui voudrait lui appliquer à la lettre le protocole de l'analyse génétique. J'ai pu m'en rendre compte avec un certain effroi, du reste, en allant moimême consulter les volumes conservés au département des Manuscrits occidentaux de la BnF... Le chercheur se heurterait d'abord aux problèmes de déchiffrement, de classement et de datation des sermons rencontrés par les éditeurs du XIXe siècle, et il serait sans doute tenté de s'en tenir à l'édition Urbain-Levesque, qui ne constitue pourtant ni une édition diplomatique, ni même une édition critique, puisqu'elle n'indique pas toutes les variantes des manuscrits. En outre, il achopperait sur une autre difficulté, qui tient cette fois à la constitution de séries : non seulement la version finale d'un sermon donné est à jamais perdue, puisque orale, mais il n'en existe jamais qu'un seul « avant-dire », Bossuet n'ayant conservé que le dernier des brouillons (pour autant que celui-ci ait été précédé par d'autres). Il paraît impossible, dans ces conditions, d'étudier le devenir d'un sermon, quelle que soit par ailleurs la perspective adoptée (stylistique, thématique, argumentative, etc.). Certes, il existe bien deux moyens de contourner cette difficulté, qui consistent à tirer parti pour l'un des reprises et des remplois opérés par Bossuet (c'est ce qu'a fait Anne Régent-Susini dans une étude remarquable ${ }^{2}$ ), et pour l'autre des sermons saisis au pied de la chaire par des tachygraphes (il en existe dix

2. Anne Régent-Susini, «Bossuet sermonnaire : une étude génétique », dans Patrick Dandrey (dir.), Génétique matérielle, génétique virtuelle, Laval (Québec), Presses universitaires de Laval, 2009, p. 71-95. 


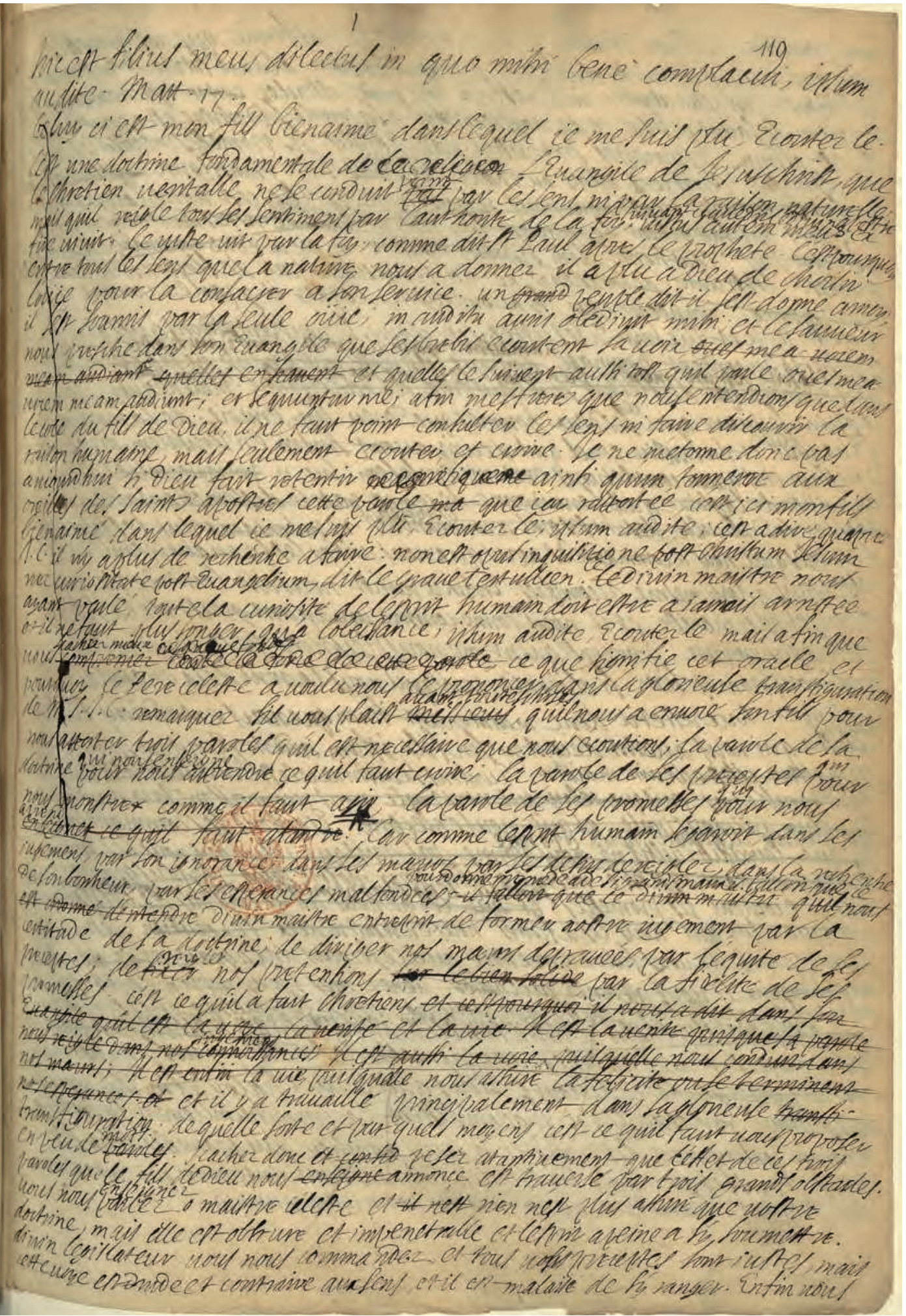

Fig. 2 : Bossuet, Sermon sur la soumission due à la parole de Jésus-Christ, Carême des Minimes, 1660, f. 119 ro (BnF, Fonds français 12822) 
dans le cas de Bossuet, et on dispose pour six d'entre eux du brouillon préparatoire du sermon) : dans les deux cas, toutefois, les séries ainsi constituées sont en nombre limité et ne représentent donc qu'une part infime des manuscrits. Davantage, en mettant uniquement en rapport des textes, elles incitent à évacuer complètement la dimension orale de la pratique discursive de Bossuet, qui fait pourtant sa spécificité : il y avait là un écueil que je souhaitais éviter.

\section{Comment avez-vous fait pour éviter ce tropisme textuel?}

J'ai tenu compte d'une série de remarques méthodologiques formulées par Almuth Grésillon dès $1993^{3}$ : réfléchissant à l'extension éventuelle de l'enquête génétique aux dossiers antérieurs à 1800, elle avait insisté sur la radicale altérité du cadre culturel dans lequel ils s'inscrivent, et suggéré de tirer parti de tous les documents exogénétiques pour mieux saisir la spécificité des pratiques scripturaires des auteurs d'Ancien Régime. Ainsi, pour être en mesure de jeter un regard neuf sur les manuscrits de Bossuet, sur lesquels je n'avais pas l'emprise philologique de mes prédécesseurs, j'ai entrepris de mener une enquête à partir de tous les textes relatifs à la pratique prédicative que j'ai pu identifier - rhétoriques de la chaire, manuels de prédication, préfaces à des recueils sermonnaires, biographies de prédicateurs -, et regardé quelle place y était accordée à l'écriture. L'enquête m'a permis de mettre au jour deux méthodes concurrentes de composition des sermons au XVII ${ }^{\mathrm{e}}$ siècle, qui impliquent à la fois des usages de l'écriture et des conceptions du sermon différents. La première, d'origine rhétorique, est tournée vers l'improvisation et ne recourt que de façon restreinte à l'écrit : le prédicateur se contente de saisir sur le papier un plan et des éléments qu'il mobilise au moment même de la performance pour produire oralement son sermon, qui est donc conçu comme un dispositif. La seconde, conseillée à l'origine aux prédicateurs débutants, mais qui tend à l'emporter sur la première au cours du siècle, est tournée vers la mémorisation et la récitation au mot à mot : le prédicateur rédige dans ce cas avec soin son sermon, qu'il envisage donc davantage comme un texte. On notera toutefois que ces deux méthodes ne sont pas exclusives l'une de l'autre, puisque certains prédicateurs conseillent de rédiger et d'apprendre par cœur le début du sermon - l'entrée en matière et l'exorde - pour en improviser en chaire le corps sur la base d'un canevas.

\section{Quelle est la méthode appliquée par Bossuet?}

Il n'en privilégie aucune, mais les applique toutes, et cela tout au long de sa carrière : on trouve dans ses manuscrits tous les cas de figure décrits dans les documents exogénétiques, du simple plan au texte entièrement rédigé et corrigé dans le détail, en passant par des sermons dont l'avant-propos, l'exorde ou une partie du développement est rédigé, alors que le reste du manuscrit demeure à l'état de notes. En outre, on remarque que les manuscrits partiellement ou pas du tout rédigés n'en constituent pas moins pour Bossuet des sermons à part entière, puisqu'il a pris le soin de les archiver et d'en constituer, lors de la préparation du Carême du Louvre, des analyses, les « sommaires », pour en faciliter la reprise partielle.

Si je vous suis bien, les manuscrits partiellement rédigés ou à l'état de notes montrent que Bossuet improvisait largement ses sermons : mais qu'en est-il dès lors de ceux qui sont entièrement rédigés? Le soin avec lequel Bossuet les corrigeait ne plaide-t-il pas en faveur d'une mémorisation et d'une récitation au mot à mot?

C'est ce que suggèrent en effet Constance CagnatDebœuf, Christine Noille et Anne Régent-Susini4 dans des études sensibles au travail stylistique fourni

\footnotetext{
3. Almuth Grésillon, « Mise au net : une critique génétique sans brouillons ? ", Paragraphes, $\mathrm{n}^{\circ}$ 9, «Les voies de l'invention aux $\mathrm{XVI}^{\mathrm{e}}$ et XVII ${ }^{\mathrm{e}}$ siècles. Études génétiques », dir. Bernard Beugnot et Robert Melançon, 1993, p. 227-232. Voir aussi «Qu'est-ce que la génétique ? Aide-mémoire et propositions théoriques », dans P. Dandrey, Génétique matérielle, génétique virtuelle, op. cit., p. 11-17.

4. Constance Cagnat-Debœuf, «L'oserais-je dire ? Timidités et audaces de Bossuet prédicateur », dans G. Ferreyrolles (dir.), Bossuet. Le Verbe et l' Histoire (1704-2004). Actes du colloque international de Paris et de Meaux, pour le troisième centenaire de la mort de Bossuet, Paris, Honoré Champion, 2006, p. 217-229 ; Christine Noille, « À la recherche du texte écrit : enquête rhétorique sur les Sermons de Bossuet », dans G. Peureux (dir.), Lectures de Bossuet. Le Carême du Louvre, Rennes, PUR, 2001, p. 89-109; Anne RégentSusini, « Bossuet sermonnaire : une étude génétique », op. cit.
} 


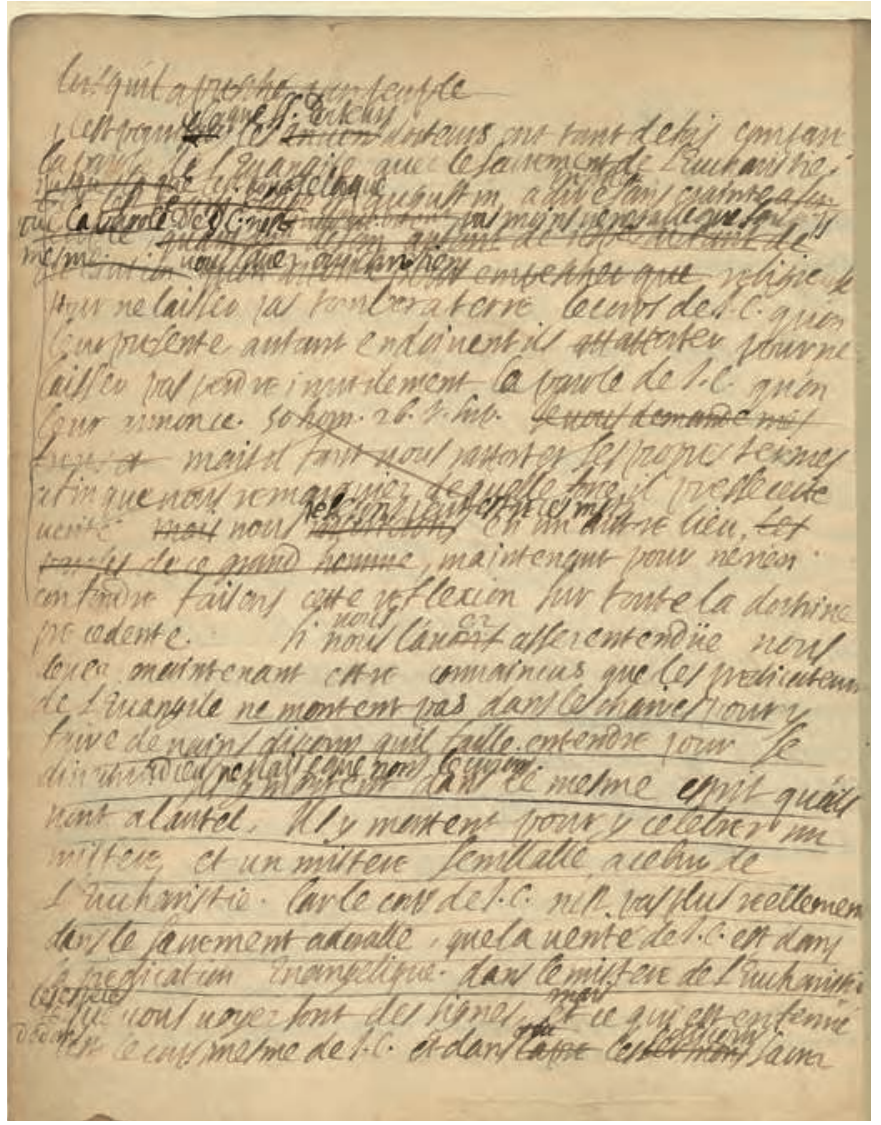

Fig. 3 : Bossuet, Sermon sur la parole de Dieu, Carême des Carmélites, 1661, f. 137 vo (BnF, Fonds français 12822)

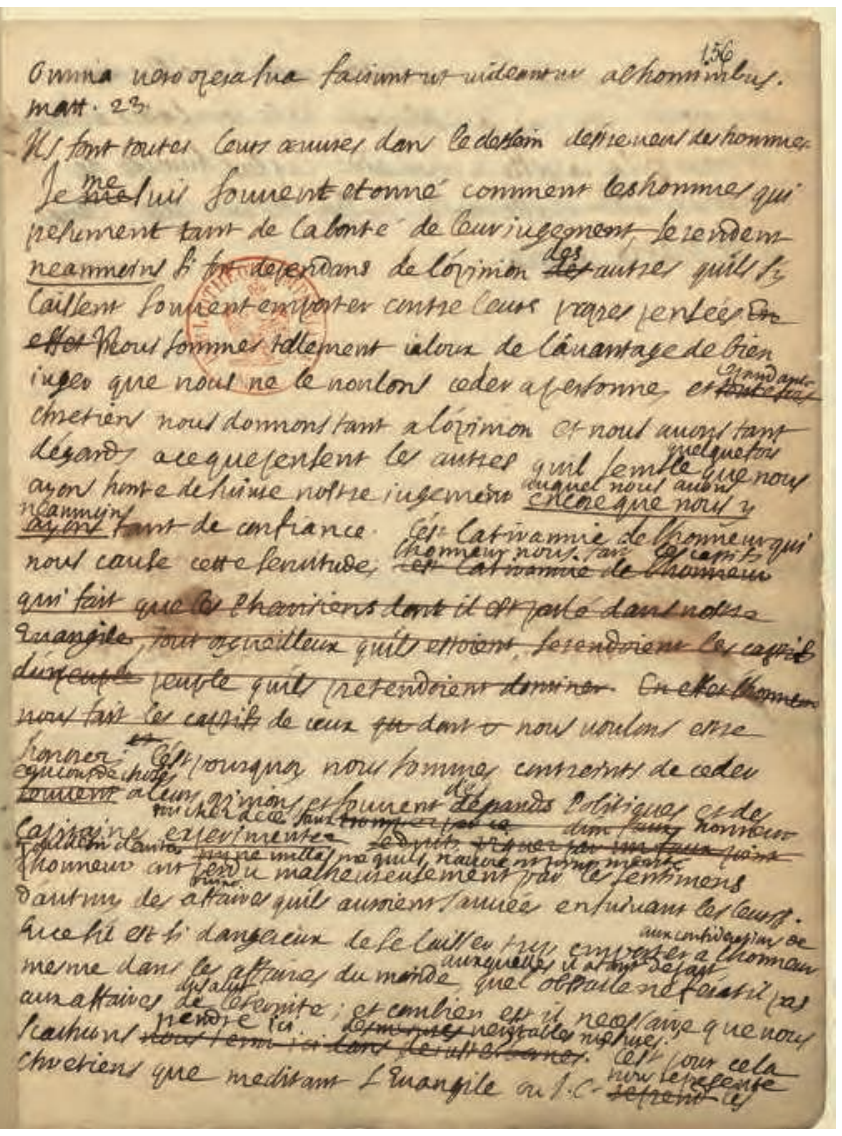

Fig. 4 : Bossuet, Sermon sur l'honneur, Carême du Louvre, 1662, f. 156 ro (BnF, Fonds français 12822) par Bossuet : on ne comprend pas bien, en particulier, pourquoi le prédicateur mettrait tant de soin à recopier en les améliorant les passages de sermons antérieurs, si ce n'est pour les apprendre par cœur ; les stations royales d'avent et de carême exigeraient une solennité qui exclurait toute improvisation. L'argument paraît recevable, mais il n'est pas sans poser plusieurs difficultés : comment expliquer dès lors que certains des sermons composés pour ces stations soient inachevés ? Parce que Bossuet aurait manqué de temps ? Mais dans ce cas, comment aurait-il eu le temps de composer et de mémoriser un nouveau sermon trois fois par semaine, lorsque l'on sait que la durée moyenne d'une prédication est d'environ une heure ? Les documents exogénétiques que j'ai consultés montrent que les prédicateurs du XVII ${ }^{e}$ siècle ne recourent plus aux techniques mnémoniques qui étaient peut-être en cours au Moyen Âge (la mémorisation par lieu), et qu'il ne faut donc pas surestimer leurs capacités mémorielles. Il m'a paru alors intéressant de tirer parti des sermons pris à l'audition que je mentionnais tout à l'heure : pour six d'entre eux, on possède en effet le brouillon préparatoire de Bossuet, et on peut donc comparer les deux versions.

\section{Et quel est le bilan de ce travail de comparaison?}

Dans deux cas, les deux versions du sermon sont à peu près identiques, à quelques détails près : en examinant attentivement ces détails, on se rend pourtant vite compte qu'ils résultent moins des erreurs de récitation de Bossuet ou de transcription du tachygraphe que des fautes de lecture d'un copiste - le temps me manque ici pour en faire la démonstration. De fait, il ne s'agit pas là de sermons pris à l'audition, mais de copies faites au XVIII ${ }^{\mathrm{e}}$ siècle sur des copies du manuscrit de Bossuet conservées alors à la bibliothèque du séminaire de SaintSulpice et aujourd'hui disparues. Dans les quatre autres cas, les différences entre sermon pris à l'audition et sermon manuscrit sont beaucoup plus importantes : Bossuet concentre ses efforts de mémorisation sur l'avant-propos et l'exorde, qu'il récite assez fidèlement, puis il improvise le corps du sermon selon le cadre 
qu'il s'est fixé d'avance ; il s'appuie en effet sur une structure argumentative - indiquée dans la division et le dessein, qui figurent à la fin de l'exorde - et des éléments discursifs - thèmes, citations scripturaires ou patristiques, expressions, figures - déterminés lors de la rédaction du manuscrit.

Reste que si l'on admet avec vous que Bossuet improvise la majeure partie de ses sermons, on ne comprend toujours pas pourquoi il prend le soin non seulement de les rédiger, mais surtout de les corriger. Comment expliquez-vous cet état de fait?

Par le fait que la fonction du manuscrit ne se limite pas à la préparation du sermon. Certes, en écrivant, Bossuet se prépare bien à prêcher : il détermine la matière et l'organisation de son discours et exerce également à l'écrit une capacité expressive qu'il mobilisera ensuite à l'oral pour formuler en chaire son sermon - en cela, sa pratique reprend les principes d'apprentissage alors à l'œuvre dans les collèges jésuites. Mais il fait plus : en écrivant, il enregistre le travail qu'il a fourni en vue d'une prédication ultérieure (soit qu'il reprenne son sermon à l'identique, soit qu'il en transpose des parties dans un nouveau sermon) ou en vue d'une diffusion non plus orale, mais écrite. Comme l'attestent les fausses copies prise à l'audition et une copie allographe conservée parmi ses manuscrits, il arrive à Bossuet de faire faire des copies de ses sermons qu'il envoie ensuite à des congrégations religieuses ou à des particuliers. Enfin, on peut supposer qu'il a songé à faire un jour imprimer ses sermons, une fois sa carrière en chaire achevée : le fait est courant parmi les prédicateurs au XVII siècle. C'est ce qui expliquerait le soin avec lequel il a rédigé, corrigé et classé par avent et par carême (le format usuel de publication des sermons) ce qu'il envisageait alors aussi comme des textes à publier. Il a toutefois abandonné ce projet en cours de carrière au profit de publications liées à ses engagements ultérieurs dans la controverse, le préceptorat et l'exégèse. Les manuscrits des sermons sont donc des objets complexes, rédigés à la fois pour être dits et pour être lus, à la fois subordonnés à et indépendants de l'événement liturgique qui en suscite la rédaction. Le processus d'autonomisation qu'ils opèrent sous nos yeux explique qu'ils peuvent être les objets non seulement de l'histoire des pratiques religieuses, mais de l'histoire et de la critique littéraires. 